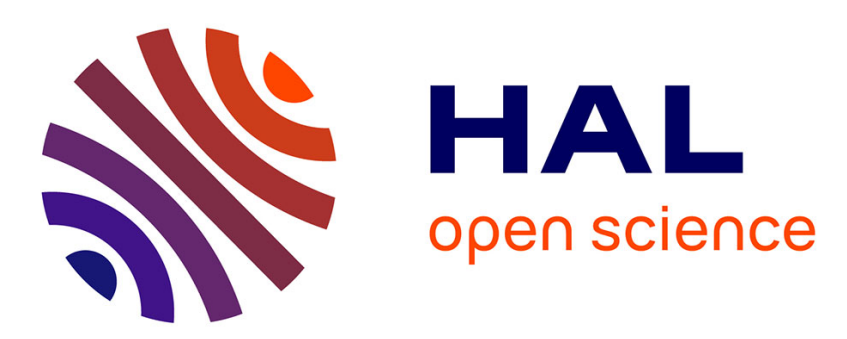

\title{
Contribution à l'étude de l'absorption à basse température des ions chromate et bichromate
}

Bernard Butowiez

\section{To cite this version:}

Bernard Butowiez. Contribution à l'étude de l'absorption à basse température des ions chromate et bichromate. Journal de Physique, 1970, 31 (5-6), pp.477-487. 10.1051/jphys:01970003105-6047700 . jpa-00206929

\section{HAL Id: jpa-00206929 https://hal.science/jpa-00206929}

Submitted on 1 Jan 1970

HAL is a multi-disciplinary open access archive for the deposit and dissemination of scientific research documents, whether they are published or not. The documents may come from teaching and research institutions in France or abroad, or from public or private research centers.
L'archive ouverte pluridisciplinaire HAL, est destinée au dépôt et à la diffusion de documents scientifiques de niveau recherche, publiés ou non, émanant des établissements d'enseignement et de recherche français ou étrangers, des laboratoires publics ou privés. 


\title{
CONTRIBUTION A L'ÉTUdE DE L'ABSORPTION A BASSE TEMPÉRATURE DES IONS CHROMATE ET BICHROMATE
}

\author{
par Bernard BUTOWIEZ (*) \\ Laboratoire de Physique M. P. 1, Faculté des Sciences, place Victor-Hugo, Marseille (B. d. Rh.)
}

(Rę̧u le 12 mai 1969, révisé le 26 janvier 1970)

\begin{abstract}
Résumé. - L'étude expérimentale de l'absorption optique à basse température des ions chromate et bichromate est reprise et complétée. L'application d'une pression hydrostatique ou d'une compression uniaxiale, en modifiant légèrement le champ cristallin, a pour effet de modifier la fréquence des raies d'absorption. Ce phénomène a été utilisé pour classer les spectres observés. Enfin, l'utilisation d'un spectrographe Jarrell-Ash (dispersion 2,4 $\AA / \mathrm{mm}$ ) a permis de mettre en évidence une structure dans les raies d'absorption de l'ion bichromate.

Dans le cas de l'ion chromate, les résultats obtenus sont confrontés aux études théoriques antérieures et l'application de la théorie des groupes permet d'expliquer qualitativement la levée de dégénérescence du premier état excité de symétrie $T_{1}$ par le champ cristallin.
\end{abstract}

Abstract. - The experimental study of optical absorption at low temperature of the chromate and dichromate ions is now taken up again. The application of a hydrostatic pressure or uniaxial compression, by slightly perturbing the crystalline field, changes the frequences of the absorption lines. This effect has been used to class the observed spectra. At last, by using a Jarrell-Ash spectrograph (dispersion $2,4 \AA / \mathrm{mm}$ ) we saw a structure in the absorption bands of the dichromate ion.

In the case of the chromate ion, the obtained results are compared to the previous theoretical studies and the application of group theoretical methods, qualitatively explains how the crystalline field removes the degeneracy and splits up the first excited state with symmetry $T_{1}$.

I. Introduction. - De nombreux travaux expérimentaux ont été consacrés à la série isoélectronique des ions $\mathrm{MnO}_{4}^{-}, \mathrm{CrO}_{4}^{--}$et $\mathrm{VO}_{4}^{---}$qui sont caractérisés par une forte absorption dans le visible ou le proche ultraviolet $[1,2]$. D'autre part, l'ion $\mathrm{Cr}_{2} \mathrm{O}_{7}^{--}$ étant réalisé à l'aide de deux tétraèdres $\mathrm{CrO}_{4}^{--}$partageant un sommet commun, les spectres d'absorption de ces ions doivent présenter des analogies.

Dès 1928 I. Obreimow et W. J. De Haas [3] avaient montré que si, à la température ordinaire, la bande d'absorption du bichromate de potassium est continue, à la température de l'hydrogène liquide elle donne naissance, du côté des grandes longueurs d'onde, à un spectre de raies fines. Cette découverte a été ensuite approfondie par J. Teltow [4] puis, par N. K. Bel'Skii et Ch. K. Mukhtarov [5]. Une étude de l'absorption ultraviolette des chromate et bichromate de potassium a d'autre part été faite par Schaumann [6].

Les études théoriques sont également nombreuses depuis le travail de Wolfsberg st Helmholz [7], mais aucune des interprétations proposées ne semble tout à fait convaincante. Il a donc semblé intéressant de reprendre une étude systématique de l'absorption des

$\left(^{*}\right)$ Cet article recouvre en partie la thèse de Doctorat d'Etat ès sciences physiques, soutenue par l'auteur à la Faculté des Sciences de Marseille, le 31 octobre 1969 et enregistrée au C. N. R. S. sous le $n^{\circ}$ A. O. 3678. ions chromate et bichromate, dans le double but de classer ces spectres et d'apporter des confirmations expérimentales à la solution théorique de ce problème.

II. Etude expérimentale. - Le montage optique utilisé est classique. La lumière émise par la source est condensée sur l'échantillon, une lentille formant l'image de cet échantillon sur la fente d'entrée du spectrographe. Un prisme de Wollaston sert de polariseur. Deux spectrographes ont été utilisés :

- un spectrographe Huet Type AII à deux prismes (ouverture $f / 8$, dispersion $45 \AA / \mathrm{mm}$ autour de $5500 \AA$ ), - un spectrographe Jarrell-Ash type Ebert Mark IV à réseau plan de 1180 traits $/ \mathrm{mm}$ (ouverture $f / 35$ dispersion $2,4 \AA / \mathrm{mm}$ dans le $1^{\text {er }}$ ordre).

Le cryostat utilisé a été décrit par P. Pesteil et R. Philip [8] et les températures inférieures à $4,2^{\circ} \mathrm{K}$ ont été obtenues par pompage sur l'hélium liquide. Après disparition du liquide réfrigérant, l'échantillon se réchauffe lentement ce qui permet, les temps de pose étant brefs, une étude qualitative de l'influence de la température.

L'étude de l'influence de la pression est plus délicate car les appareils générateurs de hautes pressions sont encombrants et ne peuvent être introduits dans un cryostat. Dans le domaine des pressions hydrostatiques, de très nombreuses expériences ont été faites 
par Drickamer et Coll. [9] et en particulier, l'influence de la pression sur la position de la première bande d'absorption des ions $\mathrm{MnO}_{4}^{-}$et $\mathrm{CrO}_{4}^{--}$a été étudiée à la température ordinaire dans le domaine 0 - $140 \mathrm{kbar}$ [10]. Dans le domaine des contraintes uniaxiales, si de nombreuses expériences ont été faites à basse température [11, 12], les cryostats décrits [13] ne peuvent être utilisés dans le cas des cristaux minces. Des appareils ont donc été conçus permettant de faire des études à basse température, le cristal étant soumis soit à une pression hydrostatique, soit à une compression uniaxiale.

- Etude sous pression hydrostatique. - La pression hydrostatique a été réalisée dans une bombe à glace décrite par Lazarev et Kan [14, 15] et modifiée en vue de son utilisation pour des études optiques [16, 17]. Ce dispositif présente l'inconvénient de ne pas permettre de suivre le déplacement continu des raies en fonction de la pression exercée et, dans les régions où les raies sont resserrées, il peut en résulter des ambiguïtés quant à l'origine d'une raie qui a été déplacée [18].

- Etude SOUS COMPRESSION UNIAXIALE. - L'application d'une pression hydrostatique, dans la mesure où elle ne modifie pas la symétrie cristalline ne peut pas lever la dégénérescence des niveaux d'énergie, elle ne peut qu'accentuer celles qui sont déjà levées. Au contraire, l'application d'une contrainte uniaxiale peut modifier la symétrie cristalline. D'autre part, il est nécessaire de relier les déplacements observés aux contraintes appliquées. Un cryostat a donc été conçu permettant de telles études [19].

Enfin, la mesure des longueurs d'onde a été faite par comparaison avec les raies d'émission d'un arc au fer en utilisant un comparateur Beaudouin. La précision des mesures dépend du spectrographe utilisé et de la finesse et de la netteté des raies. Dans le cas du spectrographe Jarrell-Ash, pour les raies les plus fines et les plus nettes, cette précision est de l'ordre de quelques centièmes d'angströms soit, pour le domaine visible, de l'ordre de $0,1 \mathrm{~cm}^{-1}$. Il est à noter que les écarts entre les composantes de structure d'une même raie sont quant à eux, déterminés avec une précision supérieure (par élimination des erreurs systématiques). Dans les meilleurs cas, elle est de $0,01 \AA$ soit quelques centièmes de $\mathrm{cm}^{-1}$. Dans le cas du spectrographe Huet, la précision est de l'ordre de $1 \mathrm{~cm}^{-1}$ pour les raies les plus nettes et les fines.

Les monocristaux ont été préparés par cristallisation lente à partir d'une solution aqueuse du sel correspondant $(*)$.

Les monocristaux ont été orientés par rapport à leurs faces et les paramètres définissant la maille cristalline, sont extraits des recueils de P. Groth [20]

(*) Quelques monocristaux de divers bichromates m'ont été fournis par le Centre de Cristallographie de Montpellier. ou de R. W. G. Wyckoff [21]. Les structures de $\mathrm{K}_{2} \mathrm{CrO}_{4}$ [22] et $\left(\mathrm{NH}_{4}\right)_{2} \mathrm{CrO}_{4}$ [23] ont été déterminées. Les ions $\mathrm{CrO}_{4}^{--}$sont tétraédriques, l'atome $\mathrm{Cr}$ occupant le centre du tétraèdre, mais à l'état solide les tétraèdres sont déformés. On peut donc à priori s'attendre à ce que la dégénérescence des niveaux d'énergie de l'ion libre tétraédrique soit levée dans le cristal. Les structures de $\mathrm{Na}_{2} \mathrm{Cr}_{2} \mathrm{O}_{7}, 2 \mathrm{H}_{2} \mathrm{O}$ [24] et $\left(\mathrm{NH}_{4}\right)_{2} \mathrm{Cr}_{2} \mathrm{O}_{7}$ [25] n'ont pas été étudiées dans le détail, mais il apparaît que l'ion $\mathrm{Cr}_{2} \mathrm{O}_{7}^{--}$est constitué à l'aide de deux tétraèdres $\mathrm{CrO}_{4}^{-}$partageant un sommet commun, occupé par un atome d'oxygène. Le bichromate de potassium existe sous 3 formes cristallines [26], la forme stable à la température ordinaire étant triclinique. La structure cristalline de cette forme est encore discutée et la présence d'un centre de symétrie est controversée [27]. Une détermination précise et récente [28] semble cependant créditer le groupe spatial $P \overline{1}$ et confirme les hypothèses faites $[29,30]$ sur l'existence de plusieurs catégories d'ions dans le cristal.

III. Résultats. - 1. Chromates - Dans le cas du chromate d'ammonium, les lames monocristallines obtenues se sont révélées être perpendiculaires à la bissectrice de l'angle aigu des axes optiques. Le spectre d'absorption à $2^{\circ} \mathrm{K}[31]$ est représenté sur la figure 1 ,
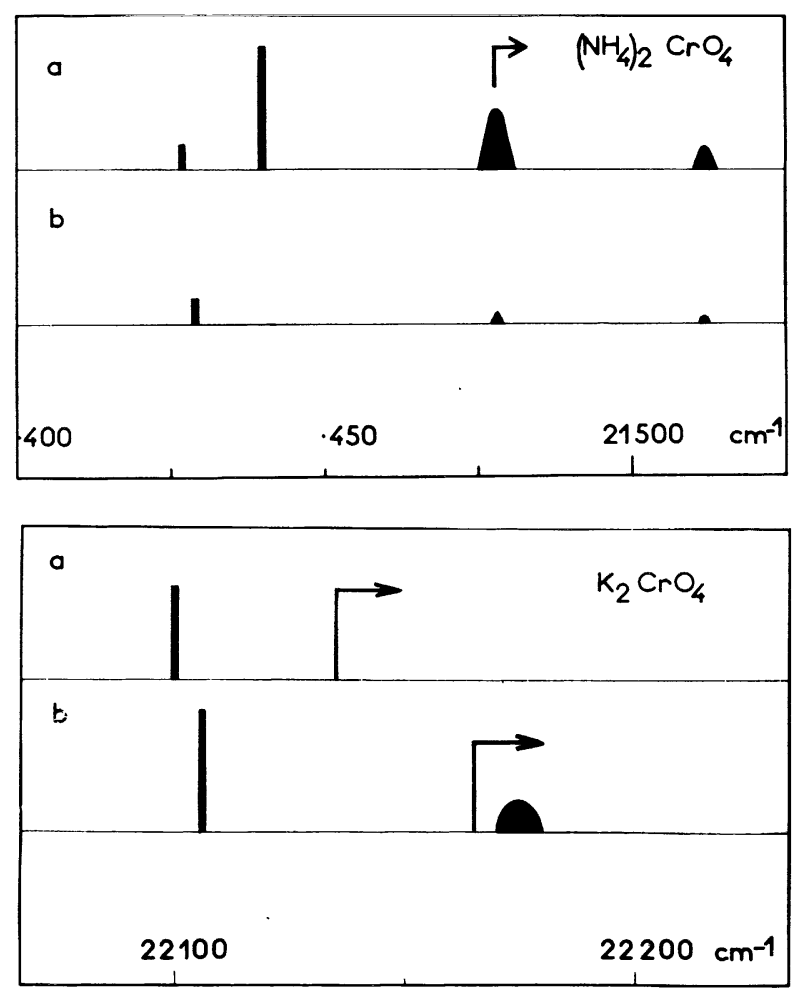

Frg. 1. - Spectres d'absorption des chromates d'ammonium et de potassium à $2^{\circ} \mathrm{K}$ en lumière polarisée rectilignement. a) vibration lumineuse parallèle à la normale optique.

b) - - dans le plan des axes optiques (parallèle à la bissectrice de l'angle obtus des axes optiques pour $\left.\left(\mathrm{NH}_{4}\right)_{2} \mathrm{CrO}_{4}\right)$.

La hauteur des raies représente leur contraste et la flèche marque le début d'une absorption continue intensifiée. 
les fréquences étant relevées dans le tableau I. Les trois raies nettes notées I, II et III sont attribuées à trois transitions électroniques pures depuis le niveau fondamental (noté 0) sur trois niveaux excités I, II et III, les niveaux I et II étant distants de $2 \mathrm{~cm}^{-1}$ seulement. J'attribue ces trois niveaux proches à la levée de dégénérescence par le champ cristallin d'un niveau qui, dans l'ion libre tétraédrique, serait triplement dégénéré. Une structure vibrationnelle très simple faisant intervenir des fréquences de l'ordre de $35 \mathrm{~cm}^{-1}$, que j'attribue à des vibrations réticulaires, est liée aux transitions précédentes. Observé à l'aide du spectrographe Jarrell-Ash, les raies fines sont élargies et ne montrent aucune structure. L'écart entre les raies I et II est mis en évidence de façon nette. La structure vibrationnelle n'apparaît plus.

\section{TABLEAU I}

\begin{tabular}{|c|c|c|c|c|}
\hline \multirow{3}{*}{$\begin{array}{l}\text { Sym- } \\
\text { boles } \\
-\end{array}$} & \multirow{2}{*}{$\begin{array}{l}\text { Fréquences } \\
\left(\mathrm{cm}^{-1}\right)\end{array}$} & \multicolumn{2}{|c|}{ Force-aspect } & \multirow{2}{*}{$\begin{array}{l}\text { Attri- } \\
\text { butions }\end{array}$} \\
\hline & & $a$ & $b$ & \\
\hline & \multicolumn{4}{|c|}{$\left(\mathrm{NH}_{4}\right)_{2} \mathrm{CrO}_{4}$} \\
\hline I & 21427 & & - & $0-\mathrm{I}$ \\
\hline II & 21429 & 一 & $\mathrm{f}$ & $0-$ II \\
\hline III & 21441 & FF & - & $0-$ III \\
\hline & 21478 & m.L.d & ff. d & III +37 \\
\hline & 21478 & C & - & III + 37 \\
\hline & 2151 & $\begin{array}{l}\text { f.L.d } \\
\mathrm{K}_{2} \mathrm{CrO}_{4}\end{array}$ & ff. $d$ & $\mathrm{III}+2 \times 3$ \\
\hline I & 22100 & $\mathrm{~F}$ & - & $0-I$ \\
\hline III & 22106 & - & $\mathrm{FF}$ & $0-$ III \\
\hline & 135 & $\mathrm{C}$ & - & $I+35$ \\
\hline & & - & $\mathrm{C}$ & III + 59 \\
\hline & 175 & - & m.L & III $+2 \times 3$ \\
\hline
\end{tabular}

Fréquences d'absorption des chromates d'ammonium et de potassium à $2^{\circ} \mathrm{K}$ en lumière polarisée rectilignement.

a) Vibration lumineuse parallèle à la normale optique.

b) Vibration lumineuse dans le plan des axes optiques (parallèle à la bissectrice de l'angle obtus des axes optiques pour $\left.\left(\mathrm{NH}_{4}\right)_{2} \mathrm{CrO}_{4}\right)$.

La force des raies est évaluée visuellement et notée : $\mathrm{FF}$, très forte ; $\mathrm{F}$, forte ; $\mathrm{m}$, moyenne ; $\mathrm{f}$, faible ; $\mathrm{ff}$, très faible ; $\mathrm{L}$, large ; $\mathrm{d}$, diffus ; $\mathrm{c}$, marque le début d'une absorption continue intensifiée.

Dans le cas du chromate de potassium, les lames monocristallines ont été taillées parallèlement à la face $q^{\prime}$ du cristal [20]. Le spectre d'absorption à $2{ }^{\circ} \mathrm{K}$ se réduit à deux raies fines notées I et III [31] que j'attribue à des transitions électroniques pures et à une structure vibrationnelle très simple faisant intervenir des fréquences de 35 et $59 \mathrm{~cm}^{-1}$. A forte dispersion, la structure vibrationnelle n'apparaît plus. Quant aux transitions électroniques, elles sont simplement élargies sans qu'il soit possible là encore d'y déceler la moindre structure. Sous l'influence d'une compression uniaxiale appliquée perpendiculairement à la face $q^{\prime}$ du cristal, la raie I se dédouble en deux composantes notées I et II et la position de ces raies en fonction de la pression exercée est représentée sur la figure 2 .

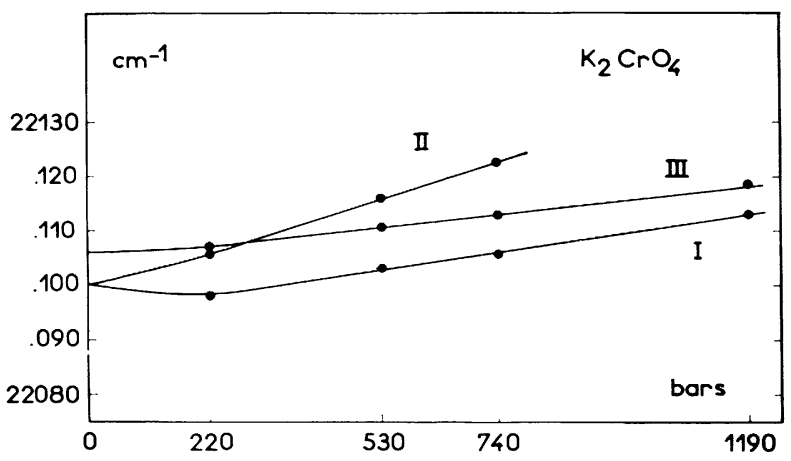

FIG. 2. - Influence d'une compression uniaxiale sur la position des raies d'absorption de $\mathrm{K}_{2} \mathrm{CrO}_{4}$ à $2^{\circ} \mathrm{K}$. La contrainte est appliquée perpendiculairement à la face $q^{\prime}$ telle qu'elle est définie par P. Groth (Chemische Kristallographie).

2. Bichromates. - a) Bichromate de potassium. Par analogie avec les notations de Teltow [4] j'appellerai respectivement $\alpha, \beta$ et $\gamma$ les directions de la bissectrice de l'angle obtus des axes optiques, de la normale optique et de la bissectrice de l'angle aigu des axes. Deux catégories de lames ont été utilisées : ces lames notées $\gamma$ et $\alpha$ ont des faces qui sont respectivement perpendiculaires aux directions $\gamma$ et $\alpha$.

Le spectre d'absorption photographié à l'aide du spectrographe Huet est représenté sur la figure $3 a$ et les fréquences d'absorption sont reportées dans le tableau II $a$. Observé à l'aide du spectrographe Jarrell-

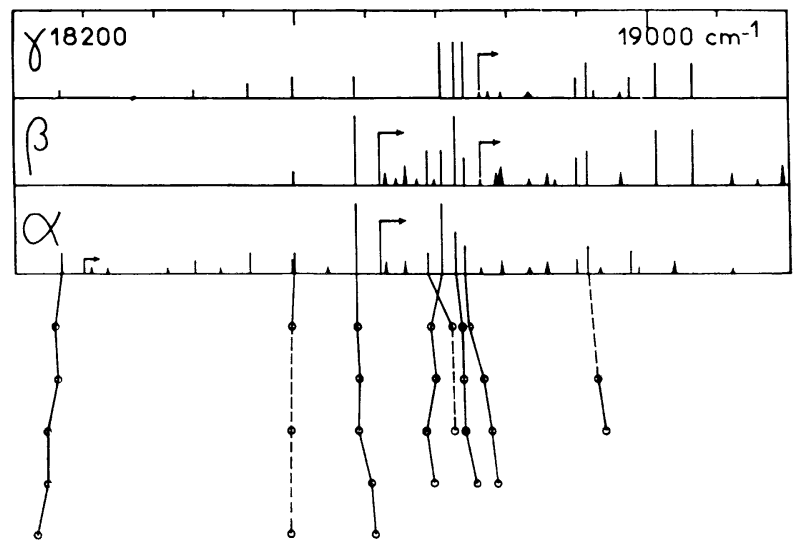

Fig. 3a. - Spectre d'absorption du bichromate de potassium à $4,2^{\circ} \mathrm{K}$ en lumière polarisée rectilignement observé à l'aide du spectrographe HUET Type AII.

- A la pression atmosphérique :

$\alpha$ ) vibration lumineuse parallèle à la bissectrice de l'angle obtus des axes optiques.

$\beta$ ) vibration lumineuse parallèle à la normale optique.

$\gamma)$ vibration lumineuse parallèle à la bissectrice de l'angle aigu des axes optiques. $\mapsto$ marque le début d'une absorption continue intensifiée et la hauteur des raies représente leur contraste. - A une pression de l'ordre de 1,7 kbar. On a figuré les déplacements de la première raie de chaque série pour cinq expériences. Les points expérimentaux sont entourés d'un cercle. 
TABleAu II $a$

Absorption à 1 atm.

\begin{tabular}{|c|c|c|c|c|c|c|c|}
\hline Symboles & Fréq. $\left(\mathrm{cm}^{-1}\right)$ & Aspect & Fréq. & Dépl. & Fréq. & Dépl. & Attributions \\
\hline \multirow[t]{9}{*}{ I $\ldots \ldots$} & 18164 & - & 18154 & -10 & 18141 & -23 & $0-\mathrm{I}$ \\
\hline & 18197 & c & - & - & - & - & $(I+33)$ \\
\hline & 18208 & d & - & - & - & - & $(I+44)$ \\
\hline & 18229 & d & - & - & - & - & $(I+2 \times 33)$ \\
\hline & 18316 & d & - & - & - & - & $(\mathrm{I}+152)$ \\
\hline & 18355 & - & 18345 & -10 & - & - & I + 191 \\
\hline & 18390 & d & 18380 & -10 & - & - & $I+191+33$ \\
\hline & 18432 & - & 18422 & -10 & 18409 & -23 & $I+268$ \\
\hline & 18492 & - & 18482 & -10 & 18468 & -24 & $I+328$ \\
\hline \multirow[t]{2}{*}{ II $\ldots \ldots$. } & 18496 & - & 18491 & -5 & 18488 & -8 & $0-$ II \\
\hline & 18542 & d & - & - & - & - & $\begin{array}{c}(\mathrm{I}+191+152+33) \\
(\mathrm{II}+46)\end{array}$ \\
\hline \multirow[t]{6}{*}{ III $\ldots \ldots$} & 18583 & - & 18583 & 0 & 18583 & 0 & $0-$ III \\
\hline & 18618 & c & 18618 & 0 & 18618 & 0 & III +35 \\
\hline & 18626 & d & 18626 & 0 & 18625 & -1 & III +43 \\
\hline & 18639 & d & 18638 & -1 & - & - & III +56 \\
\hline & 18652 & d & 18652 & 0 & 一 & - & III + $2 \times 35$ \\
\hline & 18670 & d & - & - & - & - & $(\mathrm{III}+2 \times 43)$ \\
\hline \multirow{2}{*}{ IV $\ldots}$. & 18684 & - & 18716 & +32 & 18719 & +35 & $0-$ IV \\
\hline & 18694 & d & - & - & - & - & $(\mathrm{III}+2 \times 35+43)$ \\
\hline $\mathbf{V} \ldots \ldots$ & 18706 & 一 & 18688 & -18 & 18682 & -24 & $0-\mathrm{V}$ \\
\hline VI $\ldots \ldots$ & 18726 & 一 & 18734 & +8 & 18737 & +11 & $0-\mathrm{VI}$ \\
\hline \multirow[t]{9}{*}{ VII $\ldots}$. & 18737 & - & 18743 & +6 & 18771 & +34 & $0-\mathrm{VII}$ \\
\hline & 18759 & c & 18768 & +9 & 一 & - & $\mathrm{VI}+33$ \\
\hline & 18759 & $\mathrm{~d}$ & - & - & - & - & $(\mathrm{VI}+33)$ \\
\hline & 18772 & $\mathrm{~d}$ & 一 & - & - & - & $(\mathrm{VII}+35)$ \\
\hline & $\begin{array}{l}18782 \\
18790\end{array}$ & $\mathrm{~d}$ & - & - & 18798 & +12 & $\begin{array}{c}\mathrm{VI}+56 \\
\mathrm{VI}+2 \times 33\end{array}$ \\
\hline & 18829 & dd & - & - & - & - & $(\mathrm{VII}+35+56)$ \\
\hline & 18856 & $\mathrm{~d}$ & - & - & 18857 & +1 & III +273 \\
\hline & 18867 & $\mathrm{~d}$ & - & - & 18878 & +11 & $\mathrm{VI}+141$ \\
\hline & 18897 & - & - & - & 18909 & +12 & $\mathrm{VI}+171$ \\
\hline \multirow[t]{12}{*}{ VIII $\ldots}$. & 18912 & - & - & - & 18935 & +23 & $0-$ VIII \\
\hline & 18923 & - & - & - & - & - & $(\mathrm{VII}+186)$ \\
\hline & 18929 & d & - & - & - & - & $(V+190+33)$ \\
\hline & 18960 & $\mathrm{~d}$ & - & - & - & - & $\begin{array}{c}(\mathrm{VII}+186+35) \\
(\mathrm{VIII}+48)\end{array}$ \\
\hline & 18973 & - & - & - & - & - & $V+267$ \\
\hline & 18984 & - & - & - & - & - & $(V+190+2 \times 44)$ \\
\hline & 19009 & - & - & - & 19021 & +12 & $\mathrm{VI}+171+2 \times 56$ \\
\hline & 19035 & d & - & - & - & - & $(V+329)$ \\
\hline & 19062 & - & - & - & 19074 & +12 & $\mathrm{VI}+171+3 \times 56$ \\
\hline & 19117 & d & - & - & - & - & $(\mathrm{VI}+171+4 \times 56)$ \\
\hline & 19154 & d & - & - & - & - & - \\
\hline & 19189 & d & - & - & - & - & - \\
\hline
\end{tabular}

Expériences réalisées

dans la bombe à glace 
TABleau II $b$

Force

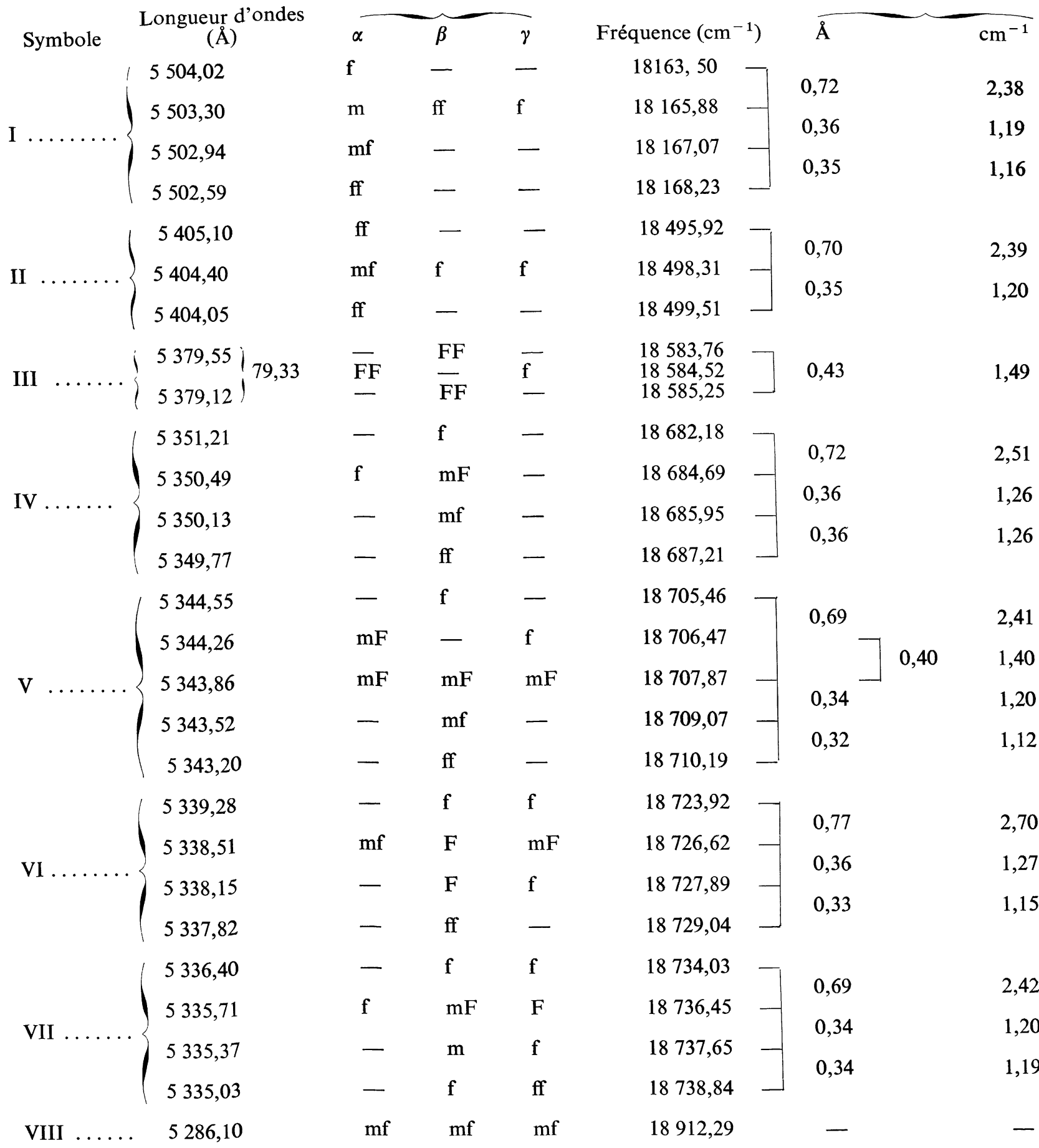

Fréquences d'absorption des transitions électroniques pures dans le bichromate de potassium à $2 \mathrm{o}$ en lumière polarisée rectilignement. L'utilisation du spectrographe Jarrell-Ash (dispersion $2,4 \AA / \mathrm{mm})$ met en évidence l'éclatement des transitions en plusieurs composantes.

๙) Vibration lumineuse parallèle à la bissectrice de l'angle obtus des axes optiques.

ß) Vibration lumineuse parallèle à la normale optique.

$\gamma)$ Vibration lumineuse parallèle à la bissectrice de l'angle aigu des axes optiques.

La force des raies est évaluée visuellement et notée : FF, très forte; F, forte ; $\mathrm{mF}$, moyennement forte ; m, moyenne ; mf, moyennement faible ; f, faible ; ff, très faible. 
Ash [32], le spectre d'absorption du bichromate de potassium présente un aspect que l'on peut résumer par les deux caractéristiques suivantes :

i) Certaines raies notées I, II, ..., VIII dans le tableau II $a$ et que je serai amené à attribuer (effet de la pression) à des transitions électroniques pures restent très fines et très nettes mais elles sont éclatées en plusieurs composantes régulièrement espacées.

ii) Les autres raies que je serai de même amené à attribuer à des raies vibrationnelles sont élargies sans qu'il soit possible d'y déceler la moindre structure. Elles sont de ce fait peu contrastées et, à l'exception des deux premières de fréquences 18355 et $18432 \mathrm{~cm}^{-1}$ n'apparaissent que très peu. La structure des raies est schématisée sur la figure $3 b$, les fréquences étant reportées dans le tableau II $b$.

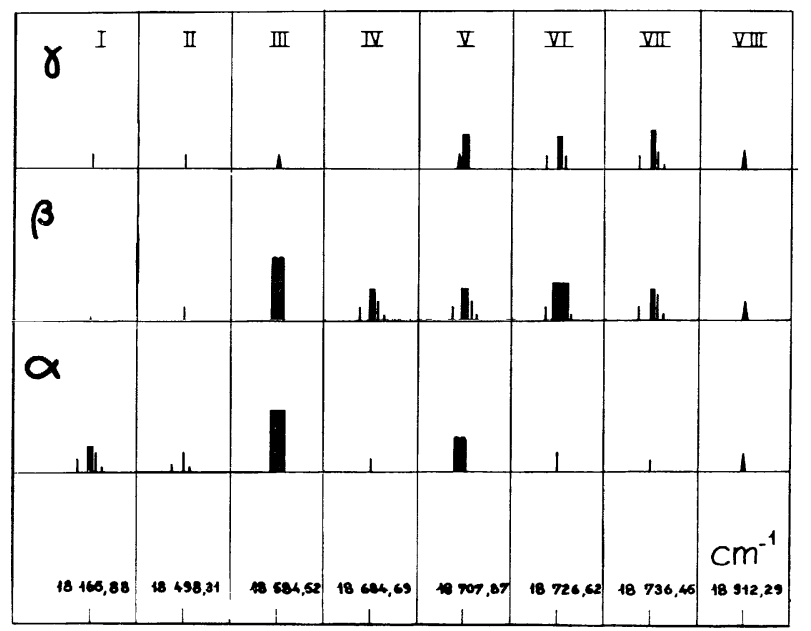

FIG. $3 b$. - Structure des transitions électroniques pures observées dans le bichromate de potassium à $2^{\circ} \mathrm{K}$, en lumière polarisée rectilignement, à l'aide du spectrographe Jarrell-Ash Ebert Mark IV.

La structure de chaque transition est représentée à une certaine échelle, sans qu'il soit possible de respecter cette échelle pour les écarts séparant les diverses transitions.

La désignation des composantes $\alpha, \beta$ et $\gamma$ est identique à celle de la figure $3 a$.

Influence d'une compression hydrostatique: cette étude a été faite sur les lames $\gamma$. L'expérience montre que les raies d'absorption restent fines, mais sont déplacées sous l'influence d'une compression hydrostatique. Les nombreuses expériences faites peuvent être résumées en deux expériences reportées dans le tableau II $a$. Ces résultats ont permis de classer les raies d'absorption en huit groupes : chaque groupe étant constitué par les raies ayant subi le même déplacement sous l'influence de la pression, ce déplacement variant d'ailleurs d'une expérience à l'autre.

Sur la figure $3 a$, j'ai représenté les déplacements de la première raie de chacun des huit groupes et ceci pour 5 expériences.

Interprétation: J'attribue la première raie de chaque groupe à une transition électronique pure et les autres raies du groupe à des transitions vibroélectroniques. Cette identification repose donc sur l'hypothèse que les fréquences vibrationnelles ne sont pas affectées par la compression hydrostatique relativement modérée exercée et donc, que les raies vibrationnelles se déplacent comme la transition électronique pure à laquelle elles sont liées. Cette hypothèse étant faite, ces expériences permettent donc d'attribuer les raies dont le déplacement a pu être mesuré à certaines transitions vibroélectroniques. Les attributions déduites sont reportées dans la dernière colonne du tableau II $a$.

Le spectre de raies de ce bichromate est donc constitué de huit transitions électroniques pures et d'une structure vibrationnelle, mais il est à noter que les fréquences vibrationnelles varient légèrement d'une série à l'autre. Cette dispersion des fréquences vibrationnelles a été observée également en absorption infrarouge ou par effet Raman de la solution et du cristal [29, 33] et a été expliquée par l'existence de plusieurs catégories d'ions dans le cristal [29, 30] hypothèse qui a été confirmée par une détermination précise de la structure du bichromate de potassium. J. K. Brandon et I. D. Brown [28] ont en effet montré que deux catégories d'ions existent dans le cristal qui diffèrent par leur géométrie.

Les fréquences vibrationnelles à l'état excité ayant des valeurs voisines de 180,270 et $330 \mathrm{~cm}^{-1}$ ont respectivement pour correspondantes les fréquences suivantes à l'état fondamental : $220 \mathrm{~cm}^{-1}$ : oscillation des 2 tétraèdres, l'un par rapport à l'autre [29]; 280-340 $\mathrm{cm}^{-1}$ : groupe de raies non attribué [33]; $365 \mathrm{~cm}^{-1}$ : vibration interne de $\mathrm{CrO}_{3}$ [33].

Les fréquences vibrationnelles de basse énergie ont également été mesurées par absorption infrarouge à $77^{\circ} \mathrm{K}$ et par effet Raman. Par effet Raman elles sont [33] : 32 - 45 - $60-75-85-96 \mathrm{~cm}^{-1}$ (raies individuelles fortes) $106-120-132-153 \mathrm{~cm}^{-1}$ (large bande groupant 4 pics).

Par absorption infrarouge elles sont [34] : 45 - 48 $66-72-79-93-116-121-138-146 \mathrm{~cm}^{-1}$, etc... On retrouve en partie ces fréquences que l'on attribue à des vibrations réticulaires et à des librations avec les valeurs suivantes à l'état excité : $33-44-56 \mathrm{~cm}^{-1}$ et $141-152 \mathrm{~cm}^{-1}$.

b) Bichromate d'ammonium. - Les lames monocristallines ont été taillées parallèlement à la face $c(0,0,1)$ du cristal telle qu'elle est définie par P. Groth [20]. Le spectre d'absorption du bichromate d'ammonium photographié à l'aide du spectrographe Jarrell-Ash [35] est représenté sur la figure 4 , les fréquences étant relevées dans le tableau III $a$. Ce spectre est constitué de 5 raies notées I, II, ..., V.

Photographiées à l'aide du spectrographe Huet [17] les deux composantes présentent un aspect identique que l'on peut résumer par les caractéristiques suivantes :

i) Les raies II et IV faibles et très fines n'apparaissent plus et la structure de la raie I n'est plus résolue. 
TABLEAU III $a$

\begin{tabular}{|c|c|c|c|c|c|c|c|}
\hline \multirow[b]{2}{*}{ Symbole } & \multirow[b]{2}{*}{ Longueur d'onde $(\AA)$} & \multicolumn{2}{|c|}{ Force } & \multirow{2}{*}{\multicolumn{2}{|c|}{ Fréquence $\left(\mathrm{cm}^{-1}\right)$}} & \multicolumn{2}{|c|}{ Ecarts } \\
\hline & & $\frac{a}{-}$ & $\underline{b}$ & & & $\AA ̊$ & $\mathrm{~cm}^{-1}$ \\
\hline \multirow{3}{*}{ I } & ( 5307,50 & $\mathrm{ff}$ & $\mathrm{ff}$ & 18836,04 & $\neg$ & \multirow{3}{*}{$\begin{array}{l}0,71 \\
0,34\end{array}$} & \multirow{3}{*}{$\begin{array}{r}2,52 \\
1,20\end{array}$} \\
\hline & 5306,79 & $\mathrm{~F}$ & F & 18838,56 & - & & \\
\hline & 5306,45 & $\mathrm{ff}$ & $\mathrm{ff}$ & 18839,76 & - & & \\
\hline II $\ldots \ldots$. & 5253,05 & f & f & 19031,28 & & & \\
\hline III . . & 5237,5 & $\mathrm{~m} \cdot \mathrm{L}$ & $\mathrm{m} . \mathrm{L}$ & 19088 & & & \\
\hline IV . & 5229,40 & ff & ff & 19117,34 & & & \\
\hline$\ldots$. & 5172,57 & mF.L & $\mathrm{mf}$ & 19327,38 & & & \\
\hline
\end{tabular}

Fréquences d'absorption des transitions électroniques pures dans le bichromate d'ammonium à $2{ }^{\circ} \mathrm{K}$ en lumière polarisée rectilignement. L'utilisation du spectrographe Jarrell-Ash met en évidence un éclatement de la raie I en 3 composantes. Les notations utilisées sont celles de la figure 6. La force des raies est évaluée visuellement et notée: $\mathrm{F}$, forte; $\mathrm{mF}$, moyennement forte ; $\mathrm{m}$, moyenne; $\mathrm{mf}$, moyennement faible; f, faible ; ff, très faible, L, large.

\section{TABLEAU III $b$}

\begin{tabular}{|c|c|c|c|c|c|c|c|c|}
\hline \multirow{4}{*}{ Symbole } & \multirow{3}{*}{$\begin{array}{c}\text { Fréquences } \\
\text { à } 1 \mathrm{~atm} .\left(\mathrm{cm}^{-1}\right) \\
-\end{array}$} & \multirow{2}{*}{\multicolumn{2}{|c|}{ Force-aspect }} & \multicolumn{2}{|c|}{ Expérience 1} & \multicolumn{2}{|c|}{ Expérience 2} & \multirow{3}{*}{ Attributions } \\
\hline & & & & fréq. & dépl. & fréq. & dépl. & \\
\hline & & \multicolumn{2}{|c|}{ - } & - & - & - & - & \\
\hline & 18838 & $\mathrm{~m}$ & fine & 18834 & -4 & 18838 & 0 & $0-I$ \\
\hline & 18862 & fff & - & - & - & - & - & +25 \\
\hline & 18888 & $\mathrm{ff}$ & - & 18884 & -4 & - & - & +50 \\
\hline & 18938 & $\mathrm{ff}$ & - & 18935 & -3 & - & - & $+2 \times 50$ \\
\hline & 18988 & fff & - & - & - & - & - & $+3 \times 50$ \\
\hline & 19041 & $\mathrm{ff}$ & - & - & - & - & - & $+4 \times 50$ \\
\hline \multirow[t]{5}{*}{ III $\ldots \ldots$} & 19088 & $\mathrm{FF}$ & $\mathrm{L}$ & 19085 & -3 & 19091 & +3 & $0-$ III \\
\hline & 19116 & fff & - & - & - & - & - & +27 \\
\hline & 19142 & f & $\mathrm{L}$ & 19139 & -3 & 19145 & +3 & +54 \\
\hline & 19195 & $\mathrm{f}$ & $\mathrm{L}$ & - & - & - & - & $+2 \times 54$ \\
\hline & 19278 & $\mathrm{ff}$ & $\mathrm{L}$ & - & - & - & - & $+4 \times 54$ \\
\hline \multirow[t]{4}{*}{$\mathrm{V}$} & 19326 & FF & fine & 19334 & +8 & 19335 & +9 & $0-\mathrm{V}$ \\
\hline & 19366 & f & - & 19373 & +7 & 19373 & +7 & +40 \\
\hline & 19482 & f & 一 & - & - & 19490 & +8 & $+4 \times 40$ \\
\hline & 19602 & $\mathrm{f}$ & - & - & - & - & - & - \\
\hline
\end{tabular}

Influence d'une compression hydrostatique sur les fréquences d'absorption du bichromate d'ammonium à $4,2 \circ \mathrm{K}$ en lumière polarisée rectilignement. La lame monocristalline est taillée parallèlement à la face $c(0,0,1)$ du cristal telle qu'elle est définie par P. Groth (Chemische Kristallographie). Les deux composantes a et b pour lesquelles la vibration lumineuse est respectivement parallèle aux axes a et b étant identiques, j'ai reporté l'absorption de l'une d'elles.

La force des raies est évaluée visuellement et notée : FF, très forte ; $\mathrm{m}$, moyenne; f, faible ; ff, très faible ; L, large.

ii) Il apparaît une structure vibrationnelle assez diffuse et peu contrastée. Les fréquences des raies correspondantes sont reportées dans le tableau III $b$.

Influence d'une compression hydrostatique: L'effet d'une compression hydrostatique sur les lames précé- dentes est résumé en les 2 expériences reportées dans le tableau III $b$. Ces expériences mettent en évidence l'existence de 3 séries de raies dans le bichromate d'ammonium (le déplacement des raies II et IV n'a pu être suivi) et j'attribue donc les cinq raies notées I, II ... V à 5 transitions électroniques pures. L'attribu- 


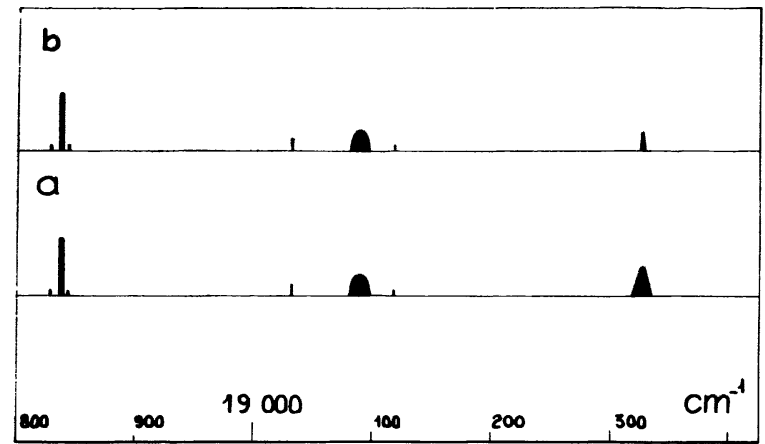

Fig. 4. - Spectre d'absorption du bichromate d'ammonium à $2^{\circ} \mathrm{K}$ en lumière polarisée rectilignement observé à l'aide du spectrographe Jarrell-Ash Ebert Mark IV.

a) Vibration lumineuse parallèle à l'axe $a$.

b) Vibration lumineuse parallèle à l'axe $b$ (normale optique).

Les notations utilisées sont extraites de P. Groth (Chemische Kristallographie).

La hauteur des raies représente leur contraste.

tion pour les raies II et IV est faite en tenant compte de leur aspect de raies faibles mais très fines. Les autres raies qui sont faibles et diffuses sont interprétées comme des transitions vibroélectroniques.
Influence de la température: L'influence de la température à la pression atmosphérique a été étudiée qualitativement en utilisant le spectrographe Huet [17]. Si le spectre n'est pratiquement pas modifié entre 4,2 et $20,4^{\circ} \mathrm{K}$, entre 20,4 et $77^{\circ} \mathrm{K}$, au fur et à mesure que la température s'élève il y a modification progressive du spectre de la façon suivante : les raies les plus faibles se brouillent et disparaissent très vite sur le fond continu. Ensuite, la raie I disparait par brouillage sans se déplacer, puis la raie III large disparaît de la même façon, mais j'ai noté un déplacement de 5 à $6 \mathrm{~cm}^{-1}$ vers les basses énergies de la raie $\mathrm{V}$ qui disparaît ensuite sur le fond continu. A $77^{\circ} \mathrm{K}$ on n'observe plus aucune structure et l'absorption est continue pour chaque composante du spectre. On voit donc que dans le cas particulier de la raie $V$, une élévation de température produit un effet inverse de celui d'une compression hydrostatique. Cet effet s'explique aisément si l'on admet que par élévation de température il y a dilatation du réseau cristallin donc diminution du champ cristallin, cet effet s'accompagnant d'une agitation plus grande qui occasionne un brouillage des raies.

c) Bichromate de sodium. - Les lames monocristallines ont été taillées parallèlement à la face $\mathrm{c}(0,0,1)$

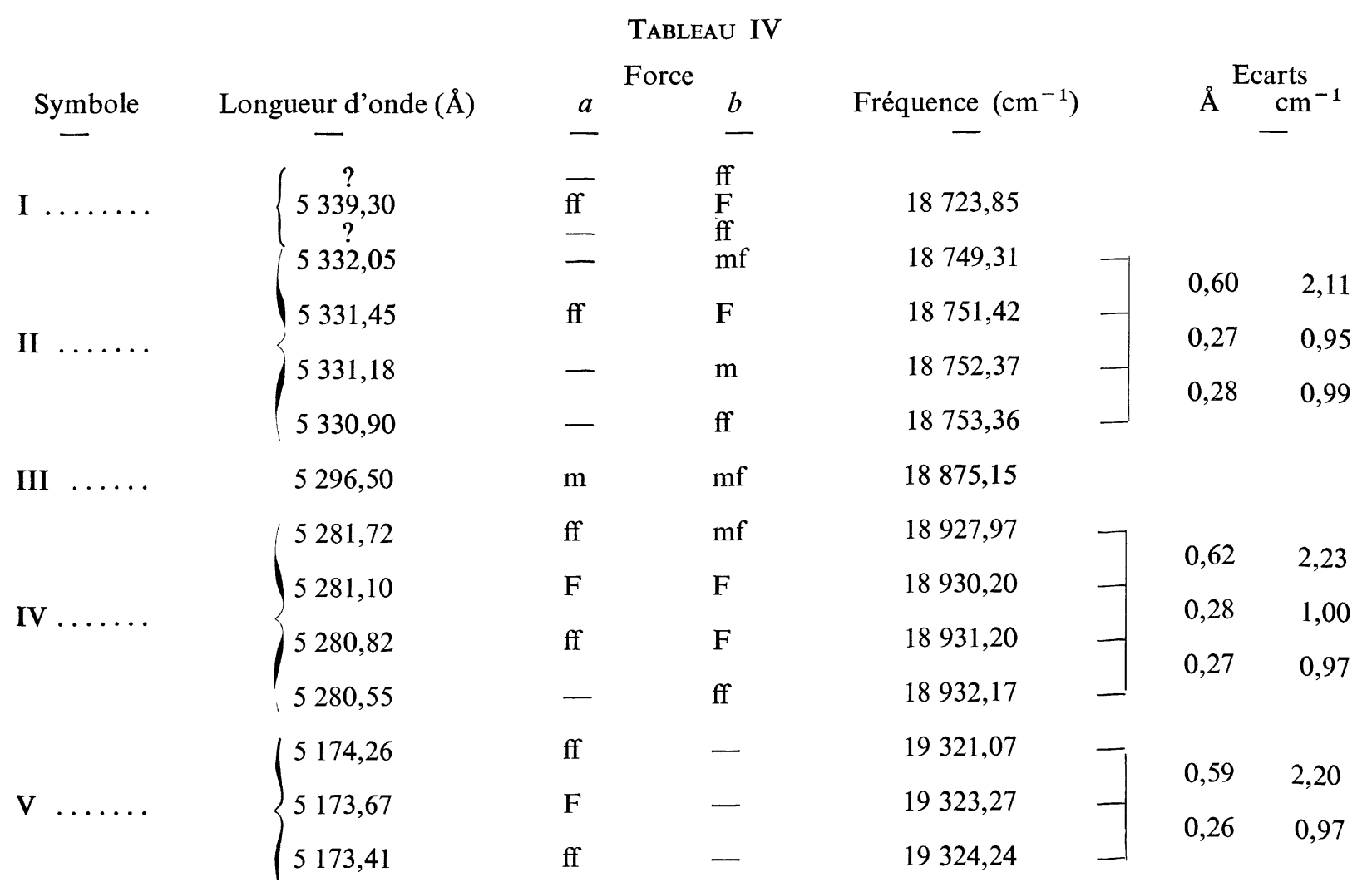

Fréquences d'absorption des transitions électroniques pures dans le bichromate de sodium à $2{ }^{\circ} \mathrm{K}$ en lumière polarisée rectilignement. L'utilisation du spectrographe Jarrell-Ash montre que chaque transition est éclatée en plusieurs composantes. Les notations utilisées sont celles de la figure 7. La force des raies est évaluée visuellement et notée : $\mathrm{F}$, forte ; $\mathrm{m}$, moyenne; $\mathrm{mf}$, moyennement faible; f, faible; ff, très faible. 
du cristal [20]. Le spectre d'absorption à $2^{\circ} \mathrm{K}$, photographié à l'aide du spectrographe Jarrell-Ash [35], est représenté sur la figure 5 , les fréquences étant reportées dans le tableau IV. Cette absorption met en évidence l'existence de 5 transitions électroniques pures notées I, II, ... V et les raies correspondantes présentent une structure nette. L'étude faite à l'aide du spectrographe Huet n'a permis de mettre en évidence que des raies vibrationnelles faibles et diffuses comparées aux transitions électroniques pures.

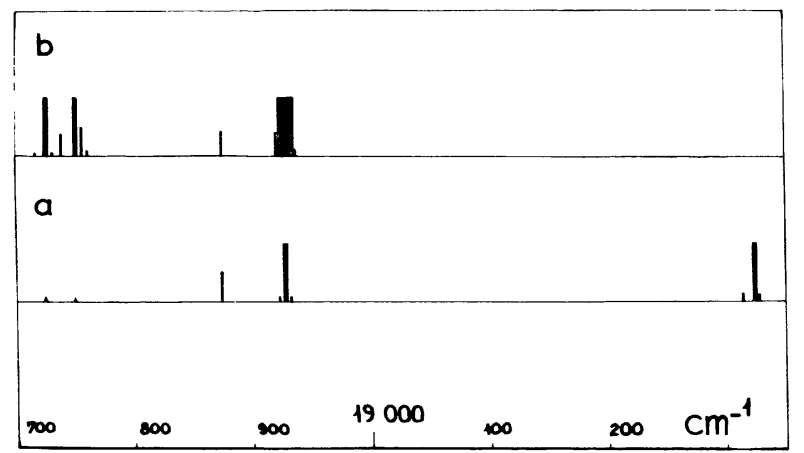

FIG. 5. - Spectre d'absorption du bichromate de sodium à $2^{\circ} \mathrm{K}$ en lumière polarisée rectilignement observé à l'aide du spectrographe Jarrell-Ash Ebert Mark IV.

a) Vibration lumineuse parallèle à l'axe $a$ (normale optique) b) Vibration lumineuse parallèle à l'axe $b$.

Les notations utilisées sont extraites de P. Groth (Chemische Kristallographie).

La structure des transitions électroniques pures est représentée à une échelle agrandie.

La hauteur des raies représente leur contraste.

IV. Discussion. - Le premier travail théorique relatif aux ions $\mathrm{MnO}_{4}^{-}$et $\mathrm{CrO}_{4}^{--}$est dû à Wolfsberg et Helmholz [7] (référence ultérieure W. H.) qui ont effectué un calcul semi-empirique des niveaux d'énergie de ces ions. Cependant, leurs résultats ne sont pas complètement satisfaisants, en particulier en ce qui concerne l'ordre des orbitales vides $3 t_{2}$ et $2 e$ qui est en contradiction avec la théorie du champ cristallin et en ce qui concerne les intensités d'absorption. Ce travail a été repris par Ballhausen et Liehr [36] (référence ultérieure $\mathrm{B}$. L.) qui sont arrivés à une conclusion inverse, l'ordre des orbitales libres étant $2 e$ et $3 t_{2}$, l'erreur de W. H. étant attribuée à une trop grande simplification des équations séculaires.

Le schéma de B. L. a été confirmé par Carrington, Schonland, Jørgensen et Coll. [37] qui montrèrent que dans l'ion $\mathrm{MnO}_{4}^{--}$l'électron célibataire occupe une orbitale $e$. Ce travail a été complété tant par l'étude des ions $\mathrm{MnO}_{4}^{--}$[38] que $\mathrm{CrO}_{4}^{---}$où les mesures montrèrent que l'électron célibataire dans $\mathrm{CrO}_{4}^{---}$ occupe de même une orbitale $e$ [39]. Fenske et Sweeney [40] montrèrent par ailleurs qu'en modifiant les paramètres de W. H., l'ordre des niveaux d'énergie était modifié, ce qui justifiait d'autres attributions. Ce travail a été ensuite repris pas Viste et Gray [41] qui confirmèrent l'ordre des niveaux de $\mathrm{B}$. L.
Ces études ont été reprises récemment surtout du point de vue théorique [42] et il était donc des plus intéressant de reprendre l'étude expérimentale de l'absorption optique de ces ions tétraédriques dans la mesure où l'identification des transitions interdites peut apporter des confirmations expérimentales aux attributions faites. Dans le schéma de B. L., la première configuration excitée résulte du passage d'un électron d'une orbitale $t_{1}$ à une orbitale $e$. Cette attribution a été confirmée par une étude de l'effet Faraday [43] et une telle excitation donne naissance à des états, de symétrie $T_{1}$ et $T_{2}$ (singulets et triplets). Dans le cas d'un système de symétrie $T_{\mathrm{d}}$, l'état fondamental étant d'autre part de symétrie $A_{1}$, la seule transition dipolaire électrique permise est la transition $A_{1} \rightarrow T_{2}$ et la première bande située à $3,25 \mathrm{eV}$ environ dans les chromates est attribuée à la transition ${ }^{1} A_{1} \rightarrow{ }^{1} T_{2}$. Les autres transitions sont interdites en l'absence de phénomène perturbateur mais pourront être observées lorsque l'ion sera par exemple inclus dans un cristal. L'ordre de ces niveaux déterminé par Ballhausen [44] est le suivant dans l'ordre des énergies croissantes :

$$
{ }^{3} T_{1} \approx{ }^{3} T_{2}<{ }^{1} T_{1}<{ }^{1} T_{2}
$$

et dans le cas des chromates le niveau ${ }^{3} T_{1}$ se situe vers $22200 \mathrm{~cm}^{-1}$ [45]. Il en résulte que l'on doit observer du côté des grandes longueurs d'ondes de la bande d'absorption des raies fines correspondant à ces niveaux et l'étude expérimentale des transitions interdites est donc intéressante.

Le spectre observé dans le cas des chromates est en concordance avec le schéma énergétique proposé par B. L. et j'attribue le groupe de trois raies fines observé dans les chromates vers $21430 \mathrm{~cm}^{-1}\left(\left(\mathrm{NH}_{4}\right)_{2} \mathrm{CrO}_{4}\right)$ et $22100 \mathrm{~cm}^{-1}\left(\mathrm{~K}_{2} \mathrm{CrO}_{4}\right)$ à la transition ${ }^{1} A_{1} \rightarrow{ }^{3} T_{1}$ depuis le niveau fondamental sur un niveau triplement dégénéré.

Tous les calculs précédents ont été faits sur l'ion $\mathrm{CrO}_{4}^{--}$supposé libre et parfaitement tétraédrique. En fait, cet ion est inclus dans un cristal et l'approximation du champ cristallin consiste à considérer l'action des ions voisins comme équivalente à un certain champ électrique interne, le champ cristallin [46]. Dans ces conditions, l'Hamiltonien de l'ion $\mathrm{CrO}_{4}^{--}$peut s'écrire $H=H_{\mathrm{L}}+V$ où $H_{\mathrm{L}}$ est l'Hamiltonien correspondant à l'ion libre et $V$ le potentiel dû à l'action des ions voisins. Ce potentiel sera supposé petit et considéré comme une perturbation de l'Hamiltonien $H_{\mathrm{L}}$.

On sait que les fonctions d'onde d'un système forment une base pour les représentations irréductibles $\mathrm{du}$ groupe de symétrie de ce système et, dans le cas où la perturbation $V$ est de symétrie moindre que celle de l'Hamiltonien $H_{\mathrm{L}}$, il y a levée de dégénérescence partielle ou totale des niveaux d'énergie de $H_{\mathrm{L}}$ [47]. La symétrie de $V$ est celle du site dans lequel est placé l'ion $\mathrm{CrO}_{4}^{--}$. Par analogie avec les perchlorates et les sulfates [1] nous admettrons que la symétrie de ce site est $C_{s}$, c'est-à-dire que de tous les éléments de symé- 
trie de l'ion $\mathrm{CrO}_{4}^{--}$, le seul qui reste est un plan de symétrie.

Dans le chromate d'ammonium, ce plan est le plan $b(0,1,0)$ tel qu'il est défini par P. Groth [20]. Les représentations irréductibles du groupe de symétrie $C_{s}$ étant unidimensionnelles $\left(A^{\prime}\right.$ et $\left.A^{\prime \prime}\right)$ il y a levée de dégénérescence totale des niveaux d'énergie primitivement classés suivant les représentations du groupe $T_{\mathrm{d}}$. La configuration excitée de symétrie $T_{1}$ donne naissance aux trois configurations suivantes: $A^{\prime \prime}, A^{\prime \prime}$ et $A^{\prime}$. Il reste donc à étudier les règles de sélection pour les transitions correspondantes $A^{\prime} \rightarrow A^{\prime}$ et $A^{\prime} \rightarrow A^{\prime \prime}$. Le potentiel $V$ étant de symétrie $C_{s}$, les méthodes de la théorie des groupes montrent immédiatement que les transitions permises sont les suivantes : $A^{\prime} \rightarrow A^{\prime}$ pour une vibration lumineuse située dans le plan de symétrie, $A^{\prime} \rightarrow A^{\prime \prime}$ pour une vibration lumineuse perpendiculaire au plan de symétrie. On doit donc observer une séparation du groupe en deux raies polarisées perpendiculairement au plan de symétrie et une raie polarisée parallèlement au plan de symétrie. Dans le cas de $\left(\mathrm{NH}_{4}\right)_{2} \mathrm{CrO}_{4}$, la normale optique étant dirigée suivant l'axe $b$ [20], on doit observer deux raies polarisées suivant la normale optique et une raie polarisée dans le plan des axes optiques, ce qui est effectivement observé. Il en est de même pour $\mathrm{K}_{2} \mathrm{CrO}_{4}$, l'ion tétraédrique étant déformé et admettant un plan de symétrie perpendiculaire à l'axe $a$, c'est-à-dire à la normale optique [22]. Ceci lève donc l'ambiguité quant à la symétrie du premier état excité. En effet, un état de symétrie $T_{2}$ se décomposant dans les mêmes conditions suivant le schéma : $T_{2} \rightarrow 2 A^{\prime}+A^{\prime \prime}$, dans cette dernière hypothèse, la polarisation des composantes $a$ et $b$ serait inversée.

V. Conclusion. - Le procédé d'investigation utilisé dans cette étude s'est donc avéré fructueux puisqu'il a permis de classer des spectres de raies qui n'avaient pu encore l'être. Le champ cristallin, considéré comme une perturbation, déplace les niveaux d'énergie de l'ion libre. En modifiant légèrement cette perturbation, la mesure des déplacements qui en résultent fournit de nombreux renseignements sur ces niveaux d'énergie.

Dans le cas des chromates, les résultats obtenus confirment des études théoriques antérieures en montrant que le premier état excité est de symétrie $T_{1}$.

Remerciements. - Je tiens à assurer M. le Professeur Pesteil qui a dirigé ces travaux après en avoir suggéré l'idée, de mes sentiments profondément reconnaissants. Je remercie également M. C. K. Jørgensen pour les discussions que nous avons eues et qui ont été pour moi très fructueuses. Enfin, mes remerciements vont également à $M$. le Professeur R. Lafont qui a bien voulu faire préparer quelques monocristaux de divers bichromates.

\section{Bibliographie}

[1] Teltow (J.), Z. Phys. Chem., 1938, B 40, 397 ; 1939, B 43, 198.

[2] Holt (S. L.) et Ballhausen (C. J.), Theoret. Chim. Acta, (Berl), 1967, 7, 313.

[3] Obreimow (I.) et De HaAs (W. J.), Communic. Phys. Lab. Univ., Leiden, 1928, $191 \mathrm{a}$.

[4] Teltow (J.), Z. Phys. Chem., 1939, B 43, 375.

[5] Bel'skit (N. K.) et Mukhtarov (Ch. K.), Optics and spectroscopy, 1963, XIV no $1,40$.

[6] Schaumann (H.), Z. für Phys., 1932, 76, 106.

[7] Wolfsberg (M.) et Helmholz (L.), J. Chem. Phys., 1952, 20,837.

[8] Pesteil (P.) et Philip (R.), Cryogenics, 1960, 1, 49.

[9] Drickamer (H. G.), Solid State Physics, 1965, 17, 1.

[10] Bentley (W. H.) et Drickamer (H. G.), J. Chem. Phys., 1961, 34, 2200.

[11] Kaplianskir (A. A.), Optics and Spectroscopy, 1959, 7, 406.

Kaplianskir (A. A.) et PrzhevuskiI (A. K.), Optics and Spectroscopy, 1966, 20, 577.

[12] Von der Osten (W.), Zeits. Phys., 1964, 174, 423. Voir également “ Colloque sur les centres colorés )) Saclay 16 mars 1967, supplément au Journal de Physique, Fas. 8-9, C4-72.

[13] Schawlow (A. L.), Piksis (A. H.) et Sugano (S.), Phys. Rev., 1961, 122, 1469.

Thomas (D. G.), J. Appl. Phys. suppl., 1961, 32, 2298.

[14] Lazarev (B. G.) et Kan (L. S.), J. Phys. Moscou, 1944, 8, 193.

[15] High Pressure Physics and Chemistry, Academic Press, 1963, I, 24 et 359.
[16] Pesteil (P.), Pesteil (L.) et Danoy (J. P.), C. $R$. Acad. Sci., 1966, 263B, 199.

[17] Butowiez (B.), C. R. Acad. Sci., 1966, 263B, 989 et 1037.

[18] Butowiez (B.), C. R. Acad. Sci., 1967, 264B, 948.

[19] Butowiez (B.) et Danoy (J. P.), Rev. de Phys. App., $1969,4,67$.

[20] Groth (P.), Chemische Kristallographie, Leipzig, 1908, II.

[21] Wyckoff (R. W. G.), Crystal Structures, Interscience publ., 1951, II.

[22] Zachariasen (W. H.) et Ziegler (G. E.), Z. Krist., 1931, 80, 164.

[23] Bujor (D.), Z. Krist., 1944, 105A, 364.

[24] Campbell (I. A.), Acta Crystal., 1956, 9, 192.

[25] Byström (A.) et Wilhelmi (K. A.), Acta Chem. Scand., 1951, 5, 1003.

[26] Jaffray (J.) et Labary (A.), C. R. Acad. Sci., 1956, 242, 1421 .

Zhukova (L. A.) et Pinsker (Z. G.), Soviet Physics, Cristallography, 1964, 9, 31.

[27] Klement (U.) et Schwab (G.), Z. Krist., 1960, 114, 170.

Rao (G. S.), J. Indian Inst. Sci. Sect., 1959, A 41, 47.

Podisko (V. S.) Tr. Inst. Krist. Akad. Nauk., S. S. S. R., 1954, 9, 327.

Parvov (V. F.) et Shubnikov (A. V.), Soviet PhysicsCristallography, 1964, 9, 361.

[28] Brandon (J. K.) et Brown (I. D.), Can. J. Chem., 1968, 46, 933. 
[29] Stammreich (H.), Bassi (D.), Sala (O.) et Siebert (H.), Spect. Acta, 1958, 13, 192.

[30] Butowiez (B.), C. R. Acad. Sci., 1967, 264B, 1212.

[31] Butowiez (B.), C. R. Acad. Sci., 1968, 267B, 1234.

[32] Butowiez (B.), C. R. Acad. Sci., 1968, 266B, 1083.

[33] LuU Dang Vinh, Reynaud (J.) et Lafont (R.), C. R. Acad. Sci., 1966, 263B, 192.

Luu Dang Vinh, Clement (C.) et Lafont (R.), C. R. Acad. Sci., 1967, 264B, 1822. Les fréquences de basse énergie m'ont été précisées par communication privée.

[34] HadNi (A.), Communication privée.

[35] Butowiez (B.), C. R. Acad. Sci., 1968, 267B, 146.

[36] Ballhausen (C. J.) et Liehr (A. D.), J. Mol. Spectrosc., 1958, 2, 342.

[37] Carrington (A.), Ingram (D. J. E.), Schonland (D.) et Symons (M. C. R.), J. Chem. Soc., 1956, 4710.

Carrington (A.) et Symons (M. C. R.), J. Chem. Soc., 1960, 889.

Schonland (D. S.), Proc. Roy. Soc., 1960, A 254, 111.

Carrington (A.) et Schonland (D. S.), Mol. Phys., $1960,3,331$.

Carrington (A.) et Jørgensen (C. K.), Mol. Phys., 1961, 4, 395.

[38] Carrington (A.), Ingram (D. J. E.), Lott (K. A. K.), Schonland (D. S.) et Symons (M. C. R.), Proc. Roy. Soc., 1960, A 254, 101.

Carrington (A.) et Symons (M. C. R.), Chem. Rev., $1963,63,443$.
Orgel (L. E.), Mol. Phys., 1964, 7, 397.

[39] Banks (E.), Greenblatt (M.) et Mc Garvey (B. R.), J. Chem. Phys., 1967, 47, 3772.

[40] Fenske (R. F.) et Sweeney (C.), Inorg. Chem., 1964, 3, 1105 .

[41] Viste (A.) et Gray (H. B.), Inorg. Chem., 1964, 3, 1113.

Basch (H.), Viste (A.) et Gray (H. B.), J. Chem. Phys., 1966, 44, 10.

[42] Jørgensen (C. K.), J. de Phys., 1965, 26, 825.

Mullen (P.), Schwochau (K.) et Jørgensen (C. K.), Chem. Phys. Let., 1969, 3, 49.

Oleari (L.), De Michelis (G.) et Di Sipio (L.), Mol. Phys., 1966, 10, 111.

Dahl (J. P.) et Johansen (H.), Theoret. Chim. Acta (Berl), 1968, 11, 8 et 26.

[43] Schatz (P. N.), Mc Caffery (A. J.), Sütaka (W.), Henning (G. N.), Ritchie (A. B.) et Stephens (P. J.), J. Chem. Phys., 1966, 45, 722.

[44] Ballhausen (C. J.), Theoret. Chim. Acta (Berl.), 1963, 1, 285.

[45] JørGensen (C. K.), Communication privée.

[46] Bethe (H. A.), Ann. Phys., 1929, 3, 133.

MoffitT (W.) et Ballhausen (C. J.), Ann. Rev. Phys., 1956, 7, 107.

Mc Clure (D. S.), Solid State Physics, 1959, 9, 399.

[47] Hamermesh (M.), Group Theory and its application to Physical Problems, Pergamon Press. 